\title{
Les clôtures symboliques des Algériennes : la virginité ou l'honneur social en question
}

\section{Barkahoum FERHATI}

\section{(2) OpenEdition}

1 Journals

\section{Édition électronique}

URL : https://journals.openedition.org/clio/6452

DOI : $10.4000 /$ clio.6452

ISSN : 1777-5299

Éditeur

Belin

\section{Édition imprimée}

Date de publication : 1 novembre 2007

Pagination : 169-180

ISBN : 978-2-85816-940-5

ISSN : 1252-7017

Référence électronique

Barkahoum FERHATI, «Les clôtures symboliques des Algériennes : la virginité ou l'honneur social en question », Clio. Femmes, Genre, Histoire [En ligne], 26 | 2007, mis en ligne le 01 janvier 2010, consulté le 22 avril 2022. URL : http://journals.openedition.org/clio/6452 ; DOI : https://doi.org/10.4000/clio. 6452

Ce document a été généré automatiquement le 22 avril 2022.

Tous droits réservés 


\title{
Les clôtures symboliques des Algériennes : la virginité ou l'honneur social en question
}

\author{
Barkahoum FERHATI
}

1 Le nouveau code de la famille algérien, dans son article 7 bis ${ }^{1}$, exige un certificat médical de santé prénuptial à produire à l'officier de l'état civil au moment de l'enregistrement du mariage ${ }^{2}$. Les officiers de l'état civil l'ont interprété en exigeant un certificat de virginité de la jeune fille. Même si les associations des femmes ${ }^{3}$ ont dénoncé ces agissements, on continue aujourd'hui d'exiger ce « certificat de la honte " ${ }^{4}$. Cette pratique par la preuve du certificat de virginité délivré par un médecin n'est pas une nouveauté en soi mais c'est son « officialisation » par des hommes de loi ou, du moins, son interprétation et le débat sur la place publique qu'il a suscité, qui nous interpellent.

2 Les raisons sociologiques, anthropologiques et théologiques de sauvegarder cette virginité sont connues. Dès longtemps, tout un arsenal de pratiques a été déployé en vue de sa conservation, de sa préservation et le cas échéant de sa réparation, coûte que coûte. Une de ces pratiques est celle liée au rbat, action de nouer, pratique secrète et intime qui procède à la "fermeture " symbolique de l'hymen de la fillette et à son " ouverture » symbolique au mariage, et qui vise à protéger la virginité sacrée des filles. Je dirai d'emblée que ce même vocable est aussi usité pour désigner un rituel aux effets maléfiques auquel les femmes recourent pour rendre "impuissant " l'homme en lui jetant des sorts pour se venger d'un amour trahi, d'une alliance non souhaitée ou encore par jalousie. Il se rapproche du rituel occidental appelé le "nouement de l'aiguillette ». Ici, il ne sera pas question de cette pratique. C'est le rbat, pratique symbolique liée aux femmes pour préserver leur virginité qui est au cœur de la présente étude.

3 Aujourd'hui cette pratique féminine, atteignant ses limites sans toutefois disparaître, se voit réinvestie par la Loi (certificat de virginité) et la Science (réparation chirurgicale de l'hymen). Nous nous trouvons là devant le renforcement, avec des méthodes 
renouvelées, de la clôture des femmes. Il s'agira ici de voir comment, pourquoi et avec quelles techniques se construit cette clôture. Je me poserai également la question des stratégies mises en place pour sortir de cet enfermement. Arrivant au certificat de virginité "exigé » aujourd'hui, je me poserai la question de ce qu'il révèle. Et je conclurai sur la résonance de cette pratique en France dans les milieux issus de l'immigration en m'interrogeant sur les limites des compromis.

Pour y parvenir, je me suis appuyée sur mes propres observations relevées autour de moi tant en Algérie qu'en France ${ }^{5}$. Je les ai confrontées avec une récente enquête que j'ai menée à l'occasion de ce travail à Bou-Saâda. Nombreux sont les travaux généraux ${ }^{6}$ sur la virginité qui permettent de comprendre ses raisons magico-religieuses, anthropologiques et ses impacts sociologiques, mais rares sont les travaux qui traitent des pratiques de la préservation de la virginité. Tandis qu'en Algérie ils sont inexistants, je noterai en Tunisie la récente étude d'Ibtissem Ben Dridi ${ }^{7}$ et au Maroc les recherches de Soumaya Naâmane-Guessous.

Pratiques et stratégies : le rbat rituel symbolique de la « fermeture » et de «l'ouverture » de l'hymen.

5 Ritualisée dans les pratiques et les comportements coutumiers, la virginité de l'Algérienne est une norme fondamentale de la société. Corps surprotégé par la famille et notamment par la mère qui veille sur la virginité de la fille car l'homme cherche toujours l'épouse vierge, "pure ", dont il se croit le seul initiateur en matière sexuelle. La virginité est ainsi une hantise pour les jeunes filles et pour les mères qui mettent en œuvre toutes sortes de stratégies pour préserver leur fille de tout contact sexuel avant le mariage et ainsi "sauver l'honneur». Il existe des pratiques traditionnelles qui consistent justement à préserver cette virginité. Celle que l'on connaît en Algérie, notamment à Bou-Saâda, est le rituel du rbat. Notons qu'il existe d'autres appellations qui participent du même principe mais qui se caractérisent par des techniques différentes d'une région à l'autre. Citons le teskar, action de fermer, le tesfah, action d'aplatir ou de blinder ${ }^{8}$. La jeune fille le subissant est dite msakra, msafha, marbouta, soit littéralement: fille fermée, fille blindée, fille ligotée ou fille nouée. La littérature occidentale du Moyen Âge nous l'a transmise sous l'appellation du rite de la ferrure. Théoriquement la fille marbouta ne peut être pénétrée par aucun homme quelle que soit sa vigueur sexuelle, et fût-elle consentante ${ }^{9}$.

6 Le rbat que nous connaissons dans la région de Bou-Saâda se pratique avant la puberté. L'âge idéal est entre huit et dix ans. Aujourd'hui, il est effectué juste avant l'entrée en première année du primaire, à six ans, tout comme la circoncision ${ }^{10}$ pour les garçons. On emmène la petite fille chez une femme spécialisée dans cette opération, la qabla, l'accoucheuse traditionnelle, l'équivalent de la sage femme. Ce rite peut être aussi accompli par la mère, une tante ou une grand-mère. Le rbat est tenu secret, car il fait partie de l'intimité des femmes. Les hommes en sont tenus à l'écart.

7 Ce rituel s'effectue par la lecture de phrases magiques et au moyen d'instruments. La phrase consacrée est répétée par la fillette sept fois de suite : wald en nas khaït wana haï que je traduis littéralement par : le fils des autres (femmes) est un fil tandis que je suis un mur. Par cette opposition fil/mur, au symbolisme sexuel évident, dont elle ne soupçonne même pas la signification, la fillette est mise en condition: désormais les hommes quels qu'ils soient représentent un danger pour elle. 
8 Pour désamorcer le pouvoir de cette formule, quelques jours avant le mariage, il suffira de l'inverser. La phrase devient alors: wald en nas haït wana khaï, que je traduis littéralement par : le fils des autres est un mur tandis que je suis un fil.

9 Ce retournement de la formule recèle un mécanisme ambivalent où, pour provoquer la fermeture, la femme est symbolisée par un mur-serrure dont aucun passe ne saurait venir à bout, alors que lorsqu'il s'agira de procéder à l'opération inverse de l'ouverture, la femme se voit dotée du pouvoir masculin de la bonne clef, symbolisée par le fil. Zine Eddine Zemmour parle de sacrifice de la fille vierge qui « est ainsi victime et en même temps source du pouvoir et objet de ce pouvoir ${ }^{11}$.

10 Des instruments tels que le métier à tisser, le cadenas, le coffre ou la poupée confectionnée à l'occasion sont utilisés comme supports symboliques dans l'accomplissement du rituel ${ }^{12}$. Dans le cas du métier à tisser, le rituel est effectué au moment de son montage. À Tlemcen, on apporte à la praticienne une aiguille et un balai. La fillette, nue, les yeux fermés, fait sept fois le tour du métier. À chaque fois elle est piquée avec une aiguille et reçoit un coup de balai. Ensuite la barre du métier est descendue et la fillette la franchit sept fois dans un sens puis sept fois dans l'autre. À Bou-Saâda, le rituel est bien plus simple. La fillette habillée enjambe le métier à tisser sept fois tout en répétant après la femme désignée pour mettre en œuvre le rituel, la formule consacrée de la fermeture.

11 Dans le cas du cadenas, on fait acheter cet objet par un garçon qui n'est pas de la famille. Il doit prendre l'argent de l'achat sans compter ni demander le prix au marchand. Ce cadenas ne doit pas être ouvert en cours de route. Pendant ce temps, la fillette est préparée: elle doit être nue et cheveux dénoués. On lui enlève boucles, épingles, colliers et bracelets. Elle doit rester debout les yeux fermés. La personne qui pratique la «fermeture » ouvre le cadenas, le place face au sexe de la fillette et le ferme avec ces paroles : « binti haït " (ma fille est un mur). Elle passe ensuite le cadenas par en dessous, vers l'arrière, l'ouvre et dit textuellement : « wald en nas khaït (le fils des autres est un fil). Par sept fois l'opération est répétée puis le cadenas est caché ou le plus souvent jeté. La veille du mariage, il faut procéder à « l'ouverture » et, pour cela, refaire l'opération dans le sens inverse.

12 Dans le cas du coffre-fort et de la serrure, on assoit sur le coffre la fillette préparée dans les mêmes conditions. On le ferme sous elle en prononçant la première formule, « ma fille est un mur... ». La veille du mariage, pour procéder à « l'ouverture», la fille est placée debout sur le coffre dont on ouvre la serrure en psalmodiant la formule inverse.

13 D'autres techniques sont utilisées, tel le marquage par le tatouage. Une légère incision est pratiquée de haut en bas sur la cuisse droite de la fillette, préparée dans les mêmes conditions, en psalmodiant les mêmes phrases magiques. Cette technique est pratiquée dans le Constantinois ${ }^{13}$ et en Tunisie ${ }^{14}$. Lors du mariage, pour procéder à "l'ouverture », on réalise une autre scarification dans le sens inverse, de bas en haut sur l'autre cuisse.

14 Et enfin, il est un autre marquage repéré chez les Reguibet, tribu de la Saoura dans le Sud algérien ${ }^{15}$. La fillette, " pour éviter tout risque d'accident ", est attachée, jambes et bras écartés, à quatre piquets fichés en terre. Une coupure est pratiquée sur le clitoris ${ }^{16}$ puis recouverte de henné. Dans tous les cas les mêmes formules sont répétées.

15 Comme si le marquage psychologique par le rbat ne se suffisait pas par lui-même, il est rendu indélébile par les marquages sur le corps des femmes, et pas seulement. Il est 
encore renforcé par l'éducation que la fillette va recevoir. En amont, les parents, la mère en particulier, auront mis le plus grand soin à la formation de la jeune fille. La prévention contre tout écart par rapport à la norme se double d'une surveillance quotidienne et d'éventuelles punitions (piment frotté sur le clitoris).

Ainsi, par la pratique du rbat, la fillette est désormais détentrice de l'honneur de tout le groupe. Elle est exclue définitivement du monde du «dehors» pour être cantonnée dans celui du « dedans ». Elle doit apprendre à faire « les choses des femmes » : cuisine, tissage et aussi apprendre à rougir et courber l'échine devant les mâles de la famille et de la société toute entière.

L'» ouverture » symbolique par le rituel du rbat la veille de la nuit de noces puis la défloration de l'hymen par l'époux sont rendues manifestes par la preuve tangible de la " chemise tachée du sang de la vierge ». Le soir même, cette chemise doit être exhibée pour être vue de tous. Certaines femmes sont même sollicitées pour expertiser le sang virginal ${ }^{17}$. Le lendemain, la famille dansera avec cette " chemise » tandis que la mariée entamera, quant à elle, une danse dite lhzam, "de la ceinture ", où s'accrochent les billets de banque qu'elle reçoit de sa belle-famille. Plus tard, cette "chemise » sera envoyée dans les familles proches et voisines, qui féliciteront la mère de la tahmirat el oujah, faire rougir le visage de la famille, ce qui s'oppose à tasfirat el oujah, faire jaunir le visage de la famille, lorsqu'il s'avère que la jeune fille n'est pas vierge. Il arrive souvent qu'on passe sur le visage des fillettes cette « chemise » pour assurer une fois de plus la protection de leur virginité. Investie de la baraka, cette chemise devient un objet sacré. Aussi est-elle gardée précieusement et ne sera-t-elle lavée que lors du premier accouchement.

Lorsque pour différentes raisons il arrive que l'affaire ne puisse être réglée, la mariée accusée de non virginité peut dans ce cas se défendre par son certificat de virginité. D'autres artifices peuvent alors être convoqués dans cette "vaste prison» des femmes. La société, aussi fermée soit-elle, offre des portes de sortie.

Les issues de secours : la clôture malléable

Lorsque le rbat est confronté à ses limites, la société des femmes - mères, grands-mères et sages femmes - se mobilise pour trouver des «issues de secours" afin de sauver l'honneur de la famille, du groupe, du clan. Comme le dit Bouhdiba : " L'histoire sociale $\mathrm{du}$ monde arabo-musulman traditionnel est une constante recherche de compensations, de fuites, de subterfuges pour contourner, dépasser le manichéisme des sexes ${ }^{18}$.

Par le passé, lorsqu'elle considérait que l'hymen n'est pas suffisamment « resserré », la qabla préparait différentes onctions pour y remédier et faciliter le saignement lors du premier rapport: "Les mariées inquiètes ont, pendant des siècles, eu recours à des artifices permettant de simuler l'hémorragie de la défloration ${ }^{19}$. Lorsque malgré tout le sang n'apparaissait pas, il ne restait plus qu'à dire que la fillette née un vendredi ne peut produire d'écoulement de sang lors du rapport. La qabla en général donnait raison à cette déclaration. Ainsi, l'honneur était sauf. Le vendredi est le jour saint chez les musulmans, le jour du pardon où il est fortement recommandé aux croyants, amis et ennemis, de se réunir dans l'enceinte de la mosquée pour la prière.

21 L'évolution et le progrès ne permettant plus aujourd'hui de faire valoir de telles causalités et d'offrir de telles issues aux égarées, d'autres tactiques de contournement sont envisagées. La pharmacie du coin est aussi appelée à la rescousse pour provoquer l'écoulement du sang. Ce sont des produits médicamenteux dont il est difficile de savoir 
par quel biais ni comment ils ont pu être repérés par les femmes. Délivrés dans le secret le plus total par le pharmacien et sans ordonnance, ces produits pharmaceutiques sont absorbés ou injectés la veille de la nuit de noces. Ici, le danger ne se mesure pas, ces femmes sont prêtes à tout.

Il est fait appel aussi à la chirurgie ${ }^{20}$ réparatrice de l'hymen par les opérations de l'hyménorrapie et de l'hyménoplastie ${ }^{21}$. Solution coûteuse, elle est pratiquée aussi bien par des chirurgiens que par des sages-femmes, les conditions d'asepsie étant en rapport avec les moyens financiers que l'on y met. Ainsi, la chirurgie donnant l'assurance de se refaire une virginité, la jeune fille peut envisager une vie sexuelle relativement libre. Sans parler du fait qu'elle peut aussi s'adonner à un simulacre de pénétration dit " petting ", flirt avancé ${ }^{22}$.

Enfin, notons la capacité de la société à défaire le rbat lorsqu'elle est forcée de constater qu'il la mène à l'impasse. En effet, il existe des situations où l'on considère que le rbat peut avoir des effets maléfiques, par exemple lorsque la jeune fille marbouta ayant dépassé l'âge du mariage, ne trouve toujours pas de mari. Pour faire affluer les demandes en mariage, il faut dénouer l'effet du rbat, avec les mêmes instruments et phrases magiques que pour «l'ouverture » à la veille du mariage. La société méprise le célibat. Le mariage est un devoir que tout musulman doit accomplir. Il est un bien précieux de Dieu. Alors qu'il était envisagé comme une protection assurant la virginité, le rbat exerce également des effets maléfiques dans le cas de l'homme dit marboute, lorsque les mauvais sorts jetés sur lui mettent en cause ses attributs virils. Il faut donc coûte que coûte l'en sortir. Dans le fond, la société qui pratique le rbat, le redoute tout autant : "Que Dieu ouvre ton nœud », est l'expression consacrée dite à tout homme et toute femme se trouvant dans une situation d'impasse.

Les salafistes, rigoristes qui prêchent tout comme les oulémas jadis ${ }^{23}$ un Islam épuré, considèrent ces pratiques comme relevant de la sorcellerie et non conformes à la religion. Ils les ont bannies. Mais la virginité demeurant à leurs yeux une valeur fondamentale, ils n'ont d'autre remède face à l'angoisse du clan que le mariage précoce afin d'échapper à la « tentation ».

On voit que, quelles qu'elles soient, les sorties de secours proposées par la société font faillite. La jeune épousée déclarée non vierge est renvoyée le soir même dans sa famille. Quant aux conséquences morales et physiques qui en découlent pour les familles, elles sont graves et peuvent aller jusqu'à l'assassinat de la fille et de la mère. Couverte de honte, la fille est bannie de la liste des jeunes prétendantes. Elle est alors destinée aux hommes âgés. Et elle pâtira avec toute sa famille de cette "honte" sur plusieurs générations.

Le passage difficile à la modernité : dilemme et ambiguïté

26 Le passage à la modernité a eu pour conséquences l'entrée des femmes dans le marché du travail et l'éclatement de la société traditionnelle et de la cellule familiale. Camille Lacoste-Dujardin dit à ce propos : «L'industrialisation du Maghreb ne semble pas avoir modifié réellement les conditions de vie des femmes... L'État légifère sur la définition des statuts individuels, sur l'éducation de même que sur la régulation des naissances... En contrepartie, l'obéissance à la doctrine islamique en tant qu'idéologie nationale contribue au maintien des croyances traditionnelles. " ${ }^{24}$ En effet, les mentalités n'ont pas suivi la marche de la modernité. Sorties du domicile familial, exposées «au danger », les femmes et leurs filles restent encore plus soumises au fardeau social de la 
virginité. Mais c'est aussi la modernité qui va leur permettre de trouver des parades et de recouvrer par la chirurgie la virginité perdue.

Ainsi, les femmes ajustent l'une à l'autre la vie moderne ayant pour conséquence une liberté sexuelle et la vie traditionnelle ayant pour préalable le maintien de la virginité comme moyen d'accès à la vie sociale. On peut s'interroger sur la résistance de cette tradition ancestrale.

Le cas des Françaises dont les parents sont issus de l'émigration notamment maghrébine et turque et qui recourent à la réparation de la virginité est à cet égard édifiant. Pourtant, les lois de la République sont là pour protéger les filles contre les archaïsmes des mentalités. Plusieurs articles publiés récemment tirent la sonnette d'alarme ${ }^{25}$. Les journalistes accablent le mouvement féministe qui a failli à sa mission en laissant pour compte cette frange des femmes à la merci « des inquisiteurs de petites vertus qui ne jurent que par l'hymen et le sang versé le soir des noces " ${ }^{26}$. Le plus frappant est le constat consternant que le recours à la chirurgie est très répandu en France. Interrogé, le Planning familial ${ }^{27}$ éprouve des difficultés à expliquer le phénomène. Il prône l'éducation pour sortir les filles du ghetto de la tradition. Quant au Conseil de l'Ordre des Médecins français, s'il interdit aux praticiens de délivrer des certificats de virginité ou de réparer l'hymen de la « honte », les médecins sollicités par les filles et parfois par leurs mères en détresse passent outre pour «sauver ces femmes ». Cette chirurgie est pourtant coûteuse, mais « elles se débrouillent, pour faire passer l'intervention pour un acte banal remboursé par la Sécurité Sociale ${ }^{28}$.

Les effets psychologiques du poids de la charge portée par les filles et leurs mères ne sont pas encore mesurés, ni ceux de la chirurgie, ni ceux de la violence de la nuit de noces, ni celui de la culpabilité qu'il faut porter sa vie durant. La psychanalyste Badia Hadj-Nasser parle « d'effet opérant dans la vie quotidienne et sociale du sujet. Il [le psychanalyste] ne peut méconnaître la résonance singulière du fait social en terme de réalité psychique sur le sujet ». ${ }^{29}$

Négociation ou compromission?

30 Alors que l'on n'a jamais vu autant de femmes occuper, dans la sphère publique, des postes de responsabilité à tous les niveaux de la hiérarchie sociale, elles continuent à rester des individus fragiles. Avec le consentement tacite des hommes, elles parviennent à trouver un compromis au péril de leur vie pour apaiser les tensions et "sauver l'honneur", un "honneur» qui résiste au changement des temps. S'agit-il tout simplement de «sauver sa peau »? L'hégémonie des hommes serait-elle toujours intacte? Faudrait-il toujours que les femmes endossent elles seules le poids de la culpabilité ? Comment en finir avec cette culpabilité ? Pourquoi la virginité offre-t-elle une telle résistance, alors que les hommes savent désormais qu'ils ne sont plus les "premiers»? Et probablement ne le seront plus jamais!

Les femmes qui s'infligent les pires violences pour apaiser les tensions sociales mais aussi pour vivre leur sexualité ne seraient-elles pas prêtes à s'assumer en tant que membres à part entière de la société ? «Les femmes maghrébines peuvent donc décliner leur vie sexuelle en de multiples nuances, selon l'éventail des moyens dont elles disposent ". ${ }^{30}$ On les enferme et elles acceptent l'enfermement dans le voile, la virginité, le mariage religieux,... Le rbat à l'instar du voile, hier moyen de conforter la société traditionnelle, serait-il le signe d'une stagnation de la société ou d'une transition obligée vers la modernité ? Puisqu'il est vrai que les femmes s'ingénient aussi 
à trouver des issues de secours sans aller jusqu'à la rupture. Jusqu'où peut-on pousser les limites de ces clôtures? Et s'il fallait rompre?

\section{BIBLIOGRAPHIE}

AINED TABET-CHELLIG Nadia, 1981, «L'Espace féminin en Algérie », Thèse de 3e cycle en sociologie, sous la dir. de Nicole Ramognino, Université de Provence Aix-Marseille1.

BELGUEDJ Mohammed Salah, 1966, Médecine traditionnelle dans le Constantinois, Strasbourg, Imprimerie Wettern.

BEN DRIDI Ibtissem, 2004, Le Tasfih en Tunisie. Un rituel de protection de la virginité féminine. Préface de Michèle Cros, Paris, L'Harmattan.

BOUHDIBA Abdelwahab, 1975, La Sexualité en Islam, Paris, PUF.

BOUSQUET Georges-Henri, 1990 (1966, 1ère ed.), L'Éthique sexuelle de l'Islam, Paris, Desclée de Brouwer.

CHEBEL Malek, 1999 Le Corps en Islam, Paris, PUF.

CURTET-POULNER Isabelle, 2007, «Pureté garantie. Hymens de complaisance », Le Nouvel Observateur, 15-21 mars 2007.

DOUTE Edmond, 1909, La société musulmane au Maghreb : Magie et religions dans l'Afrique du Nord, Alger, Jourdan.

FERHATI Barkahoum, 2006, « La lutte contre l'excision au Soudan : entre politique volontariste, mondialisation et résistance sociale », TERRA, revue en ligne, Esquisse $n^{\circ} 6$.

-, 2007 « De l'Algérie au Soudan. Des voies et des voix de la lutte contre l'excision », in AnneMarie Moulin (dir.), Les Labyrinthes du corps en Islam, Paris, CEDEJ/Karthala (à paraître).

GADANT Monique, 1991, «Le Corps dominé des femmes, réflexions sur la valeur de la virginité (Algérie) », L’Homme et la société, « Femmes et sociétés », n 99-100, 1-2, p. 37-56.

HADJ-NASSER Badia, 1989, « La Fascination de la virginité et sa résonance dans le corps des femmes immigrées », in A. Yahyaoui (dir.), Corps espace-temps et traces de l'exil, Alger, p. 45-52.

LACOSTE-DUJARDIN Camille, 1985, Des Mères contre des femmes. Maternités et patriarcat au Maghreb, Paris, La Découverte.

NAAMANE-GUESSOUS Soumaya, 1982, Au-delà de toute pudeur. Sexualité féminine au Maroc, Casablanca, 2e édition, SODEN.

TAFFY Serge, 2007, « Françaises et musulmanes. Vierges à tout prix », Nouvel Observateur, 15-21 mars 2007.

TOUALBI Nouredine, 1983, La circoncision. Blessure narcissique ou promotion sociale, Alger, ENAL.

ZEMMOUR Zine Eddine, 2002, « Jeune fille, famille et virginité. Approche anthropologique de la tradition $"$, Confluences, $n^{\circ} 41$, p. 65-76.

ZERDOUMI Nefissa, 1982, Enfants d'hier. L'éducation des enfants en milieu algérien, Paris, Maspero. 


\section{NOTES}

1.Article 7 bis de l'Ordonnance $n^{\circ} 05-02$ du 18 moharrem 1426 correspondant au 27 février 2005 modifiant et complétant la loi n 84-11 du 9 juin 1984 portant code de la famille.

2.Le texte dit : « Les futurs époux doivent présenter un document médical, datant de moins de trois (3) mois et attestant qu'ils ne sont atteints d'aucune maladie ou qu'ils ne présentent aucun facteur de risque qui contre-indique le mariage. Avant la rédaction du contrat de mariage, le notaire ou l'officier de l'état civil doit constater que les deux parties se sont soumises aux examens médicaux et ont eu connaissance des maladies ou des facteurs de risques qu'ils pourraient révéler et qui contre-indiquent le mariage. Il en est fait mention dans l'acte de mariage. Les conditions et modalités d'application de cet article seront définies par voie réglementaire ».

3. « 20 ans Barakat », « Le forum des Libertés », « Djazairouna », « Fatma Nsoumer », « Touiza », etc.

4.Liberté, La Tribune, El Watan, quotidiens algériens.

5.Ici, mes interlocutrices sont de jeunes étudiantes émigrées originaires de la région de Bou-Saâda, ma ville natale, située à $250 \mathrm{~km}$ au Sud d'Alger, connue pour être une ville arabophone.

6.Douté 1909 ; Bousquet 1990 : 103 ; Gadant 1991.

7.Je remercie Jocelyne Dakhlia et Omar Bendana pour m'avoir indiqué cette référence.

8.Belguedj $1966: 15$.

9.Chebel $1999: 80$.

10.Toualbi 1983.

11.Zemmour $2002: 72$.

12.Ained Tabet-Chellig 1981.

13.Soit l'Est algérien dont la capitale est Constantine.

14.Ben Dridi 2004.

15.Zerdoumi 1982.

16.Cette dernière coutume se rapproche de la pratique de l'excision dite sunnite telle qu'elle est pratiquée au Soudan. Le clitoris est, selon les familles, coupé ou seulement incisé. Voir à ce sujet Ferhati 2006 et Ferhati 2007.

17.Il semblerait que c'est à son odeur enivrante et à sa couleur rose pâle que le sang de la vierge est reconnu.

18.Bouhdiba 1975 : 241.

19.Naamane-Gessous $1982: 201$.

20.Dans les pays où l'on pratique l'excision et l'infibulation, la question de la virginité ne se pose même pas. Voir Ferhati 2006.

21.La première chirurgie consiste en la suture de l'hymen tandis que la deuxième consiste à décoller une partie de la muqueuse du vagin pour reconstituer l'hymen.

22.Chebel $1999: 177$.

23.Fondée en 1931 par Ibn Badis, cette organisation religieuse s'oppose aux confréries religieuses en Algérie.

24.Lacoste-Dujardin 1985 : 235.

25.Raffy $2007: 93$.

26.Curtet-Poulner $2007: 94$.

27.Curtet-Poulner $2007: 94$.

28.Curtet-Poulner 2007 : 95. 
29.Hadj-Nasser $1989: 52$.

30.Ben Dridi 2004 : 116

\section{RÉSUMÉS}

Les clôtures symboliques des Algériennes : la virginité ou l'honneur social en question. Liée à l'honneur de la famille, la virginité est une question qui a toujours hanté l'esprit des jeunes filles algériennes. Toutes sortes de pratiques sont envisagées par la société pour la préserver. La plus connue est celles du rbat (action de nouer), dit teskar (action de fermer) ou encore tesfah (action de blinder). Au moyen de techniques ritualisées, elle consiste en la «fermeture » symbolique de l'hymen avant la puberté et son "ouverture» symbolique la veille du mariage. La "chemise tachée du sang de la vierge » doit en être la preuve indéniable : elle authentifie que l'honneur de la famille, du groupe, est intact. Lorsque, pour diverses raisons, l'hymen fait défaut, la société déploie toutes sortes de stratégies palliatives. Aujourd'hui, le certificat de virginité et la reconstitution de l'hymen par la chirurgie plastique, l'hyménorrapie ou l'hyménoplastie, viennent renforcer la pratique symbolique du rabt. La question fondamentale que je pose dans cet article est : peut-on envisager de casser la clôture «malléable » de cette « vaste prison » des femmes afin que les Algériennes puissent intégrer la société en tant qu'individus et non en tant qu'emblèmes de l'honneur de la famille?

The symbolic enclosure of Algerian women: debates concerning virginity or social honour. Linked to familial honour, virginity has always haunted the minds of Algerian girls. Society has devised all manner of practices to preserve it. The best known is the rbat (to tie), known as teskar (to close) or tesfah (to wall up). By means of ritualised techniques, it consists of a symbolic "closing" of the hymen before puberty and its "opening" the day before the marriage. The "shirt marked with the virgin's blood" constitutes then the undeniable proof authenticating the intact honour of the family and the group. When, for various reasons, the hymen is missing, society deploys a range of palliative strategies. Today, a certificate of virginity and the reconstitution of the hymen by plastic surgery reinforce the practice of the rbat. This article asks whether it is possible to break the "malleable" fence of this "vast prison" for women in order to allow them to integrate society as individuals and not as emblems of the family's honour?

\section{INDEX}

Keywords : Algerian women, familial honour, ritual, virginity

Mots-clés : femmes algériennes, honneur familial, hymen, virginité, rituel

\section{AUTEUR}

\section{BARKAHOUM FERHATI}

Barkahoum FERHATI est maître de recherche au CNRPAH à Alger, chercheure associée au CHIM, EHESS. Elle est l'auteure de deux ouvrages : Le musée national Etienne Dinet de Bou-Saâda, Alger, 
INAS, 2004 et De la «tolérance » en Algérie : Enjeux en soubassement, 1830-1962, Alger, El Othmaniya, 2007, et elle a publié plusieurs articles autour des questions des femmes. Aujourd'hui, elle mène une étude comparative autour des questions du genre entre le Soudan et l'Algérie. 